\title{
Combinatorial properties of the Temperley-Lieb algebra of a Coxeter group
}

\author{
Alfonso Pesiri
}

Received: 2 November 2011 / Accepted: 19 June 2012 / Published online: 30 June 2012

(C) Springer Science+Business Media, LLC 2012

\begin{abstract}
We study two families of polynomials that play the same role in the Temperley-Lieb algebra of a Coxeter group as the Kazhdan-Lusztig and $R$ polynomials play in the Hecke algebra of the group. Our results include recursions, non-recursive formulas, symmetry properties and expressions for the constant term. We focus mainly on non-branching Coxeter graphs.
\end{abstract}

Keywords Temperley-Lieb algebra $\cdot$ Hecke algebra $\cdot$ Kazhdan-Lusztig basis . Coxeter group

\section{Introduction}

The Temperley-Lieb algebra $T L(X)$ is a quotient of the Hecke algebra $\mathscr{H}(X)$ associated to a Coxeter group $W(X), X$ being an arbitrary Coxeter graph. It first appeared in [49], in the context of statistical mechanics (see, e.g., [30]). The case $X=A$ was studied by Jones (see [31]) in connection to knot theory. For an arbitrary Coxeter graph, the Temperley-Lieb algebra was studied by Graham. More precisely, in [20] Graham showed that $T L(X)$ is finite dimensional whenever $X$ is of type $A, B, D, E, F, H$ or $I$. If $X \neq A$ then $T L(X)$ is usually referred to as the generalized Temperley-Lieb algebra.

The algebra $T L(X)$ has many properties similar to the Hecke algebra $\mathscr{H}(X)$. In particular, it is shown in [24] that $T L(X)$ inherits an involution from $\mathscr{H}(X)$ and that it always has a basis, indexed by the fully commutative elements of $W(X)$, with some

This work is part of the author's doctoral dissertation, written under the direction of Prof. F. Brenti at the University of Rome "Tor Vergata".

\footnotetext{
A. Pesiri $(\bowtie)$

Dipartimento di Matematica, Università di Roma “Tor Vergata”, Via della Ricerca Scientifica, 00133 Roma, Italy

e-mail: pesiri@mat.uniroma2.it
} 
remarkable properties, called an IC basis (see [15] and [24]). Thus, one has two families of polynomials, indexed by pairs of fully commutative elements of $W(X)$, which are analogous to the Kazhdan-Lusztig and $R$-polynomials of $\mathscr{H}(X)$. Although the Kazhdan-Lusztig and $R$-polynomials have been extensively studied (see, e.g., [2, 4, $5,7-10,12,14,16,23,28,29,34,35,39,43])$ and $T L(X)$ plays an important role in several areas and has also been extensively studied (see, e.g., [11, 17-21, 25, 31, 32, $37,41,42]$ ), these polynomials have not been investigated very much. Our purpose in this work is to begin the study of these polynomials from a combinatorial point of view. More precisely we obtain recursions, non-recursive formulas, symmetry properties and expressions for the constant terms, for these polynomials. To do this, we need to study some auxiliary polynomials (which have no analogue in $\mathscr{H}(X)$, and which in some sense express the relationship between $\mathscr{H}(X)$ and $T L(X))$ which were first defined in [24]. Most of our results hold for non-branching Coxeter graphs, although some hold in full generality. Our results show that there is a close relationship between Kazhdan-Lusztig and $R$-polynomials and their analogue in $T L(X)$.

The organization of the paper is as follows. In the next section we recall some generalities on the Hecke algebra, Kazhdan-Lusztig polynomials and the KazhdanLusztig basis for $\mathscr{H}(X)$. Moreover, we introduce the Temperley-Lieb algebra and the families of polynomials $\left\{a_{x, w}\right\}$ and $\left\{L_{x, w}\right\}$ that we study in this work. In Sect. 3 we prove our main result (Theorem 3.7) on $D$-polynomials, which holds for all finite irreducible and affine non-branching Coxeter graphs $X$ such that $X \neq \widetilde{F}_{4}$, and we obtain some explicit formulas for the $D$-polynomials in type $A$. In Sects. 4 and 5 we use Theorem 3.7 to obtain recursions, non-recursive formulas, symmetry properties and expressions for the constant term, for the polynomials $\left\{a_{x, w}\right\}$ and $\left\{L_{x, w}\right\}$.

\section{Preliminaries}

In this section we recall some basic facts about Hecke algebras $\mathscr{H}(X)$ and Temperley-Lieb algebras $T L(X), X$ being any Coxeter graph. Let $W(X)$ be the Coxeter group having $X$ as Coxeter graph and $S(X)$ as set of generators. Let $\mathscr{A}$ be the ring of Laurent polynomials $\mathbb{Z}\left[q^{\frac{1}{2}}, q^{-\frac{1}{2}}\right]$. The Hecke algebra $\mathscr{H}(X)$ associated to $W(X)$ is an $\mathscr{A}$-algebra with linear basis $\left\{T_{w}: w \in W(X)\right\}$ (see, e.g., [3, §6.1]). For all $w \in W(X)$ and $s \in S(X)$ the multiplication law is determined by

$$
T_{w} T_{s}= \begin{cases}T_{w s} & \text { if } \ell(w s)>\ell(w), \\ q T_{w s}+(q-1) T_{w} & \text { if } \ell(w s)<\ell(w),\end{cases}
$$

where $\ell$ denotes the usual length function of $W(X)$. We refer to $\left\{T_{w}: w \in W(X)\right\}$ as the $T$-basis for $\mathscr{H}(X)$.

Let $e$ be the identity element of $W(X)$. One easily checks that $T_{s}^{2}=(q-1) T_{s}+$ $q T_{e}$, being $T_{e}$ the identity element, and so $T_{s}^{-1}=q^{-1}\left(T_{s}-(q-1) T_{e}\right)$. It follows that all the elements $T_{w}$ are invertible, since, if $w=s_{1} \cdots s_{r}$ and $\ell(w)=r$, then $T_{w}=T_{s_{1}} \cdots T_{s_{r}}$. To express $T_{w}^{-1}$ as a linear combination of elements in the basis, one obtains the so-called $R$-polynomials. For a proof of the following result we refer to [27, §7.4]. 
Theorem 2.1 There is a unique family of polynomials $\left\{R_{x, w}(q)\right\}_{x, w \in W(X)} \subseteq \mathbb{Z}[q]$ such that

$$
T_{w^{-1}}^{-1}=\varepsilon_{w} q^{-\ell(w)} \sum_{x \leq w} \varepsilon_{x} R_{x, w}(q) T_{x},
$$

and $R_{x, w}(q)=0$ if $x \not \leq w$, where $\varepsilon_{x} \stackrel{\text { def }}{=}(-1)^{\ell(x)}$. Furthermore, $R_{x, w}(q)=1$ if $x=w$.

Define a map $\iota: \mathscr{H} \rightarrow \mathscr{H}$ such that $\iota\left(T_{w}\right)=\left(T_{w^{-1}}\right)^{-1}, \iota(q)=q^{-1}$ and extend by linearity. We refer the reader to $[27, \S 7.7]$ for the proof of the following result.

Proposition 2.2 The map ı is a ring homomorphism of order 2 on $\mathscr{H}(X)$.

In [33], the Kazhdan and Lusztig prove this basic theorem:

Theorem 2.3 There exists a unique basis $\left\{C_{w}: w \in W(X)\right\}$ for $\mathscr{H}(X)$ such that the following properties hold:

(i) $\iota\left(C_{w}\right)=C_{w}$,

(ii) $C_{w}=\varepsilon_{w} q^{\frac{\ell(w)}{2}} \sum_{x \leq w} \varepsilon_{x} q^{-\ell(x)} P_{x, w}\left(q^{-1}\right) T_{x}$,

where $\left\{P_{x, w}(q)\right\} \subseteq \mathbb{Z}[q], P_{w, w}(q)=1$ and $\operatorname{deg}\left(P_{x, w}(q)\right) \leq \frac{1}{2}(\ell(w)-\ell(x)-1)$ if $x<w$.

The polynomials $\left\{P_{x, w}(q)\right\}_{x, w \in W(X)}$ are the so-called Kazhdan-Lusztig polynomials of $W(X)$. In [27, §7.9] it is shown that one can substitute the basis $\left\{C_{w}: w \in\right.$ $W(X)\}$ with the equivalent basis $\left\{C_{w}^{\prime}: w \in W(X)\right\}$, where

$$
C_{w}^{\prime}=q^{-\frac{\ell(w)}{2}} \sum_{x \leq w} P_{x, w}(q) T_{x} .
$$

For the rest of this paper we will refer to the latter basis as the Kazhdan-Lusztig basis for $\mathscr{H}(X)$.

It is a routine exercise to prove the following properties (see, e.g., [3, §5, Exercises 3 and 7(a)]).

Lemma 2.4 Let $x, w \in W(X)$ be such that $x \leq w$. If $\ell(w)-\ell(x) \leq 2$ then

(i) $R_{x, w}(q)=(q-1)^{\ell(w)-\ell(x)}$;

(ii) $P_{x, w}(q)=1$.

Let $s_{i}, s_{j} \in S(X)$ and denote by $\left\langle s_{i}, s_{j}\right\rangle$ the parabolic subgroup of $W(X)$ generated by $s_{i}$ and $s_{j}$. Following [20], we consider the two-sided ideal $J(X)$ generated by all elements of $\mathscr{H}(X)$ of the form

$$
\sum_{w \in\left\langle s_{i}, s_{j}\right\rangle} T_{w},
$$

where $\left(s_{i}, s_{j}\right)$ runs over all pairs of non-commuting generators in $S(X)$ such that the order of $s_{i} s_{j}$ is finite. 
Definition 2.5 The generalized Temperley-Lieb algebra is $T L(X) \stackrel{\text { def }}{=} \mathscr{H}(X) / J(X)$.

When $X$ is of type $A$, we refer to $T L(X)$ as the Temperley-Lieb algebra. In order to describe a basis for $T L(X)$, we recall the notion of a fully commutative element for $W(X)$ (see [48]).

Definition 2.6 An element $w \in W(X)$ is fully commutative if any reduced expression for $w$ can be obtained from any other by applying Coxeter relations that involve only commuting generators. We let

$$
W_{c}(X) \stackrel{\text { def }}{=}\{w \in W(X): w \text { is a fully commutative element }\} .
$$

If $X=A_{n-1}$ then $W(X) \simeq S_{n}$ (see [3, Example 1.2.3]) and $W_{c}\left(A_{n-1}\right)$ may be described as the set of elements of $W\left(A_{n-1}\right)$ all of whose reduced expressions avoid substrings of the form $s_{i} s_{i \pm 1} s_{i}$, for all $s_{i} \in S$ (see [48, Proposition 1.1]). Another description of $W_{c}\left(A_{n-1}\right)$ may be given in terms of pattern avoidance: namely, in [1, Theorem 2.1] Billey, Jockusch and Stanley show that $W_{c}\left(A_{n-1}\right)$ coincides with the set of permutations avoiding the pattern 321. Moreover $\left|W_{c}\left(A_{n-1}\right)\right|=C_{n}$, where $C_{n}=\frac{1}{n+1}\left(\begin{array}{c}2 n \\ n\end{array}\right)$ denotes the $n$th Catalan number (see [18, Proposition 3] for further details).

Let $t_{w}=\sigma\left(T_{w}\right)$, where $\sigma: \mathscr{H} \rightarrow \mathscr{H} / J$ is the canonical projection. A proof of the following can be found in [20].

Theorem 2.7 $T L(X)$ admits an $\mathscr{A}$-basis of the form $\left\{t_{w}: w \in W_{c}(X)\right\}$.

We call $\left\{t_{w}: w \in W_{c}(X)\right\}$ the $t$-basis of $T L(X)$. By (2.1), it satisfies

$$
t_{w} t_{s}= \begin{cases}t_{w s} & \text { if } \ell(w s)>\ell(w) \\ q t_{w s}+(q-1) t_{w} & \text { if } \ell(w s)<\ell(w) .\end{cases}
$$

Observe that if $w s \notin W_{c}(X)$, then $t_{w s}$ can be expressed as linear combination of the $t$-basis elements by means of the following result (see [24, Lemma 1.5]).

Proposition 2.8 Let $w \in W(X)$. Then there exists a unique family of polynomials $\left\{D_{x, w}(q)\right\}_{x \in W_{c}(X)} \subseteq \mathbb{Z}[q]$ such that

$$
t_{w}=\sum_{\substack{x \in W_{c}(X) \\ x \leq w}} D_{x, w}(q) t_{x},
$$

where $D_{w, w}(q)=1$ if $w \in W_{c}(X)$. Furthermore, $D_{x, w}(q)=0$ if $x \not \leq w$.

From the fact that the involution $\iota$ fixes the ideal $J(X)$ (see [24, Lemma 1.4]), it follows that $\iota$ induces an involution on $T L(X)$, which we still denote by $\iota$, if there is no danger of confusion. More precisely, we have the following result.

Proposition 2.9 The map $\iota$ is a ring homomorphism of order 2 such that $\iota\left(t_{w}\right)=$ $\left(t_{w^{-1}}\right)^{-1}$ and $\iota(q)=q^{-1}$. 
To express the image of $t_{w}$ under $\iota$ as a linear combination of elements of the $t$-basis, one defines a new family of polynomials (see [24, §2]).

Proposition 2.10 Let $w \in W_{c}(X)$. Then there exists a unique family of polynomials $\left\{a_{y, w}(q)\right\} \subseteq \mathbb{Z}[q]$ such that

$$
\left(t_{w^{-1}}\right)^{-1}=q^{-\ell(w)} \sum_{\substack{y \in W_{c}(X) \\ y \leq w}} a_{y, w}(q) t_{y},
$$

where $a_{w, w}(q)=1$.

The polynomials $\left\{a_{x, w}(q)\right\}$ associated to $T L(X)$ play the same role as the polynomials $\left\{R_{x, w}(q)\right\}$ associated to $\mathscr{H}(X)$. They both represent the coordinates of elements of the form $\iota\left(t_{w}\right)$ (respectively, $\left.\iota\left(T_{w}\right)\right)$ with respect to the $t$-basis (respectively, $T$-basis).

The generalized Temperley-Lieb algebra admits a basis $\left\{c_{w}: w \in W_{c}(X)\right\}$ which is analogous to the Kazhdan-Lusztig basis $\left\{C_{w}^{\prime}: w \in W(X)\right\}$ of $\mathscr{H}(X)$. The following is a restatement of [24, Theorem 3.6].

Theorem 2.11 There exists a unique basis $\left\{c_{w}: w \in W_{c}\right\}$ of $T L(X)$ such that

(i) $\iota\left(c_{w}\right)=c_{w}$,

(ii) $c_{w}=\sum_{\substack{x \in W_{c} \\ x \leq w}} q^{-\frac{\ell(x)}{2}} L_{x, w}\left(q^{-\frac{1}{2}}\right) t_{x}$,

where $\left\{L_{x, w}\left(q^{-\frac{1}{2}}\right)\right\} \subseteq q^{-\frac{1}{2}} \mathbb{Z}\left[q^{-\frac{1}{2}}\right], L_{x, x}\left(q^{-\frac{1}{2}}\right)=1$, and $L_{x, w}\left(q^{-\frac{1}{2}}\right)=0$ if $x \not \leq w$.

This basis is often called an IC basis (see [24, §2]).

Comparing the definition of $c_{w}$ with that of $C_{w}^{\prime}$, we notice that the polynomials $L_{x, w}\left(q^{-\frac{1}{2}}\right)$ play the same role as $q^{\frac{\ell(x)-\ell(w)}{2}} P_{x, w}(q)$, where $P_{x, w}(q)$ are the KazhdanLusztig polynomials defined in Theorem 2.3. Since the Kazhdan-Lusztig basis and the IC basis are both $\iota$-invariant and since $\iota(J)=J$, it is natural to ask to what extent $\left\{\sigma\left(C_{w}^{\prime}\right): w \in W(X)\right\}$ coincides with $\left\{c_{w}: w \in W_{c}(X)\right\}$.

Definition 2.12 We say that a Coxeter graph $X$ satisfies the projection property if

$$
\left\{\sigma\left(C_{w}^{\prime}\right): w \in W_{c}(X)\right\}=\left\{c_{w}: w \in W_{c}(X)\right\} .
$$

A sufficient condition for a Coxeter graph to have the projection property is given in [25, Proposition 1.2.3].

Proposition 2.13 Let $\sigma: \mathscr{H}(X) \rightarrow \mathscr{H}(X) / J(X)=T L(X)$ be the canonical projection. If $K \operatorname{er}(\sigma)$ is spanned by the basis elements $C_{w}^{\prime}$ that it contains, then $X$ satisfies the projection property.

The kernel of the canonical projection $\sigma: \mathscr{H}\left(A_{n}\right) \rightarrow \mathscr{H}\left(A_{n}\right) / J\left(A_{n}\right)$ is spanned by all elements $C_{w}^{\prime}$ such that $w \notin W_{c}\left(A_{n}\right)$. This stems from the fact that $W_{c}\left(A_{n}\right)$ is 
a union of two-sided Kazhdan-Lusztig cells (see [19, Proposition 3.1.1]). Therefore, type $A$ has the projection property and the same argument holds for types $B$ and $I_{2}(m)$ (see [26, Theorem 3.1.1] and [22, Proposition 6.14]). This fact was also verified for types $\mathrm{H}_{3}, \mathrm{H}_{4}$, and $\mathrm{F}_{4}$, by means of computer calculations (see [26, §3]). The converse of Proposition 2.13 is not true in general. A counterexample is given in [38, Example 2.5], where Losonczy shows that type $D_{n}$, with $n \geq 4$, has the projection property, but Proposition 2.13 does not apply. Similar problems arise whenever $X$ is a Coxeter graph that contains a vertex connected to at least three other vertices. Graphs having this property are sometimes called branching graphs. Some examples of branching graphs are types $D, E_{6}, E_{7}, E_{8}$, and in these cases Proposition 2.13 does not apply (see [26, Corollary 3.1.3]). No example of a Coxeter group that fails to satisfy the projection property is known and even in type $E$ it is an open problem (see $[26, \S 2])$.

We remark that the hypothesis of Proposition 2.13 is equivalent to asking that $\sigma\left(C_{w}^{\prime}\right)=0$, for all elements $w \notin W_{c}(X)$ (see [26, Theorem 2.2.3]). In particular, one may wonder whether the map $\sigma: \mathscr{H}(X) \rightarrow \mathscr{H}(X) / J(X)$ satisfies

$$
\sigma\left(C_{w}^{\prime}\right)= \begin{cases}c_{w} & \text { if } w \in W_{c}(X), \\ 0 & \text { if } w \notin W_{c}(X) .\end{cases}
$$

The answer is affirmative in types $A, B, I_{2}(m), F_{4}, H_{3}$ and $H_{4}$, and negative for types $D, E_{6}, E_{7}$ and $E_{8}$ (for a complete discussion of these results, see [22] and [26]). More generally, if $W(X)$ is a finite irreducible or affine Coxeter group, relation (2.4) holds if and only if $W_{c}(X)$ is a union of two-sided Kazhdan-Lusztig cells (see [46, Lemma 2.4] and [26, Theorem 2.2.3]). On the other hand, in [45, §3] Shi shows that $W_{c}(X)$ is a union of two-sided Kazhdan-Lusztig cells if and only if $X$ is nonbranching and $X \neq \widetilde{F}_{4}$. We sum up these properties in the following.

Theorem 2.14 Let $X$ be a finite irreducible or affine Coxeter graph. Then, relation (2.4) holds if and only if $X$ is non-branching and $X \neq \widetilde{F}_{4}$.

\section{The polynomials $D_{x, w}$}

In this section we study the $D$-polynomials defined in Proposition 2.8. More precisely, we obtain a recurrence relation for the polynomials $\left\{D_{x, w}(q)\right\}_{x \in W_{c}(X), w \in W(X)}$, $X$ being an arbitrary Coxeter graph. Then we focus on the Coxeter graphs satisfying equation (2.4) and obtain some results concerning symmetry properties, the value of the constant term, and explicit formulas for the $D$-polynomials of some families of Bruhat intervals. From now on we will denote by $\ell(x, w)$ the number $\ell(w)-\ell(x)$, for every $x, w \in W(X)$ such that $x \leq w$.

Let $X$ be an arbitrary Coxeter graph. We begin with the following recursion for the $D$-polynomials. 
Theorem 3.1 Let $w \notin W_{c}(X)$ and $s \in S(X)$ be such that $w s \notin W_{c}(X)$, with $\ell(w s)<$ $\ell(w)$. Then, for all $x \in W_{c}(X), x \leq w$, we have

$$
D_{x, w}=\widetilde{D_{x, w}}+\sum_{\substack{y \in W_{c}(X), y s \notin W_{c}(X) \\ y s>y}} D_{x, y s} D_{y, w s},
$$

where

$$
\widetilde{D_{x, w}}= \begin{cases}D_{x s, w s}+(q-1) D_{x, w s} & \text { if } x s<x \\ q D_{x s, w s} & \text { if } x<x s \in W_{c}(X), \\ 0 & \text { if } x<x s \notin W_{c}(X) .\end{cases}
$$

Proof On the one hand, by Proposition 2.8,

$$
t_{w}=\sum_{\substack{x \in W_{c}(X) \\ x \leq w}} D_{x, w} t_{x}
$$

On the other hand, letting $v \stackrel{\text { def }}{=} w s$,

$$
\begin{aligned}
& t_{w}=t_{v} t_{s}=\left(\sum_{\substack{y \in W_{c}(X) \\
y \leq v}} D_{y, v} t_{y}\right) t_{s} \\
& =\sum_{\substack{y \in W_{c}(X) \\
y \leq v, y s>y}} D_{y, v} t_{y s}+\sum_{\substack{y \in W_{c}(X) \\
y \leq v, y s<y}} D_{y, v}\left(q t_{y s}+(q-1) t_{y}\right) \\
& =\sum_{\substack{y \in W_{c}(X), y s \in W_{c}(X) \\
y \leq v, y s>y}} D_{y, v} t_{y s}+\sum_{\substack{y \in W_{c}(X), y s \notin W_{c}(X) \\
y \leq v, y s>y}} D_{y, v} t_{y s} \\
& +\sum_{\substack{y \in W_{c}(X) \\
y \leq v, y s<y}} D_{y, v} q t_{y s}+\sum_{\substack{y \in W_{c}(X) \\
y \leq v, y s<y}}(q-1) D_{y, v} t_{y} \\
& =\sum_{\substack{y \in W_{c}(X), y s \in W_{c}(X) \\
y \leq v, y s>y}} D_{y, v} t_{y s}+\sum_{\substack{y \in W_{c}(X) \\
y \leq v, y s<y}} D_{y, v} q t_{y s} \\
& +\sum_{\substack{y \in W_{c}(X) \\
y s<y \leq v}}(q-1) D_{y, v} t_{y}+\sum_{\substack{y \in W_{c}(X), y s \notin W_{c}(X) \\
y \leq v, y s>y}} D_{y, v}\left(\sum_{\substack{x \in W_{c}(X) \\
x \leq y s}} D_{x, y s} t_{x}\right) \\
& =\sum_{\substack{x \in W_{c}(X) \\
x s<x \leq w}} D_{x s, w s} t_{x}+\sum_{\substack{x s \in W_{c}(X) \\
x \leq w, x s>x}} q D_{x s, w s} t_{x} \\
& +\sum_{\substack{x \in W_{c}(X) \\
x s<x \leq w}}(q-1) D_{x, w s} t_{x}+\sum_{x \in W_{c}(X)}\left(\sum_{\substack{y \in W_{c}(X), y s \notin W_{c}(X) \\
y<y s}} D_{x, y s} D_{y, w s}\right) t_{x}
\end{aligned}
$$


(note that $x s \in W_{c}(X), x<x s \Rightarrow x \in W_{c}(X)$ ). Extracting the coefficient of $t_{x}$ we get

$$
D_{x, w}= \begin{cases}D_{x s, w s}+(q-1) D_{x, w s}+b(x, w) & \text { if } x s<x, \\ q D_{x s, w s}+b(x, w) & \text { if } x<x s \in W_{c}(X), \\ b(x, w) & \text { if } x<x s \notin W_{c}(X),\end{cases}
$$

where

$$
b(x, w)=\sum_{y \in W_{c}(X), y s \notin W_{c}(X)} D_{x, y s} D_{y, w s},
$$

as desired.

It is interesting to note that the recursion in Theorem 3.1 is similar to the one for the parabolic Kazhdan-Lusztig polynomials (see [13]).

The preceding recursion can sometimes be solved explicitly. In the proof of the next result we need the notion of Grassmannian and bi-Grassmannian elements (see, e.g., $[36, \S 3]$ and $[3, \S 5$, Exercise 38]).

Definition 3.2 Let $w \in W\left(A_{n-1}\right)$ and define $D_{R}(w) \stackrel{\text { def }}{=}|\{s \in S(X): w s<w\}|$. The permutation $w$ is called Grassmannian if $\left|D_{R}(w)\right| \leq 1$ and bi-Grassmannian if $\left|D_{R}(w)\right|=\left|D_{R}\left(w^{-1}\right)\right|=1$.

As a consequence of [1, Theorem 2.1], if $w \in W\left(A_{n-1}\right)$ is Grassmannian then $w \in W_{c}\left(A_{n-1}\right)$.

In the sequel we will need the following properties of the Bruhat order, which are usually referred to as the Subword Property (see, e.g., [3, Theorem 2.2.2]) and the Lifting Property (see, e.g., [3, Proposition 2.2.7]).

Theorem 3.3 Subword property Let $s_{1} \cdots s_{r}$ be a reduced expression of $v \in W(X)$ and let $u \in W(X)$. Then $u \leq v$ if and only if there exists a reduced expression $s_{i_{1}} \cdots s_{i_{k}}$ of $u$ such that $1 \leq i_{1} \leq \cdots \leq i_{k} \leq r$.

Lemma 3.4 Lifting property Let $x, w \in W$ be such that $x<w$ and suppose $s \in S$ such that $w s<w$ and $x s>x$. Then $x \leq w s$ and $x s \leq w$.

Corollary 3.5 Let $X$ be of type A and let $x_{0} \in W_{c}(X)$ be a bi-Grassmannian element. If $x_{0}$ is a maximal element in the Bruhat order of $W_{c}(X)$, then $D_{x_{0}, w}(q)=\varepsilon_{x_{0}} \varepsilon_{w}$, for all elements $w \geq x_{0}$.

Proof If $w \in W_{c}(X)$ then the result is trivial. Therefore, assume $w \notin W_{c}(X)$. Observe that if $s \in S(X)$ is such that $x_{0} s>x_{0}$, then $x_{0} s \notin W_{c}(X)$. Moreover, if $x_{0} s>x_{0}$ and $y \in W_{c}(X)$ is such that $y s>x_{0}$ then $y \geq x_{0}$ by Lemma 3.4, so $y=x_{0}$. Hence

$$
\left\{y \in W_{c}(X): y s>x_{0}\right\}=\left\{x_{0}\right\},
$$

for any $s \notin D_{R}\left(x_{0}\right)$. Choosing $s$ such that $x_{0} s>x_{0}, w s<w$ (there exists such an $s$ since $x_{0}$ is a bi-Grassmannian element, while $w \notin W_{c}(X)$ is not Grassmannian), the 
third case of Theorem 3.1 applies, so

$$
D_{x_{0}, w}(q)=D_{x_{0}, x_{0} s}(q) D_{x_{0}, w s}(q) \text {. }
$$

We proceed by induction on $\ell\left(x_{0}, w\right)$. Suppose $\ell\left(x_{0}, w\right)=1$, with $w \notin W_{c}\left(A_{n-1}\right)$. By Theorem 3.3 it follows that $w$ admits a reduced expression of the form $x_{1} s_{i} s_{i+1} s_{i} x_{2}$, with $x_{1}, x_{2} \in W_{c}\left(A_{n-1}\right), s_{i}, s_{i+1} \in S\left(A_{n-1}\right)$, and $x_{0}$ admits a reduced expression of the form $x_{1} \hat{s_{i}} s_{i+1} s_{i} x_{2}$ or $x_{1} s_{i} s_{i+1} \hat{s_{i}} x_{2}$, since $x_{0} \in W_{c}\left(A_{n-1}\right)$. Therefore

$$
t_{w}=t_{x_{1}} t_{s_{i} s_{i+1} s_{i}} t_{x_{2}}=t_{x_{1}}\left(-t_{s_{i} s_{i+1}}-t_{s_{i+1} s_{i}}-t_{s_{i+1}}-t_{s_{i}}-t_{e}\right) t_{x_{2}},
$$

and the statement follows by applying (2.3). If $\ell\left(x_{0}, w\right)>1$, then

$$
D_{x_{0}, w}(q)=D_{x_{0}, x_{0} s}(q) D_{x_{0}, w s}(q)=-D_{x_{0}, w s}(q)=-\varepsilon_{x_{0}} \varepsilon_{w s}=\varepsilon_{x_{0}} \varepsilon_{w} .
$$

From here to the end of this section we will denote by $X$ a Coxeter graph satisfying (2.4). Observe that $D_{x, w}(q)=\delta_{x, w}$ if $x, w \in W_{c}(X)$.

Lemma 3.6 For all $y \in W_{c}(X)$ and $w \notin W_{c}(X)$, we have

$$
\sum_{y \leq x \leq w} D_{y, x}(q) P_{x, w}(q)=0 .
$$

Proof Let $w \in W(X)$. Then, by Proposition 2.8,

$$
\begin{aligned}
\sigma\left(C_{w}^{\prime}\right) & =q^{-\frac{\ell(w)}{2}} \sum_{x \leq w} P_{x, w}(q) \sigma\left(T_{x}\right) \\
& =q^{-\frac{\ell(w)}{2}} \sum_{x \leq w} P_{x, w}(q)\left(\sum_{\substack{y \in W_{c}(X) \\
y \leq x}} D_{y, x}(q) t_{y}\right) \\
& =q^{-\frac{\ell(w)}{2}} \sum_{\substack{y \in W_{c}(X) \\
y \leq w}}\left(\sum_{y \leq x \leq w} D_{y, x}(q) P_{x, w}(q)\right) t_{y} .
\end{aligned}
$$

When $w \notin W_{c}(X)$ we get $\sigma\left(C_{w}^{\prime}\right)=0$, so the expression in round brackets must vanish and the statement follows.

The following is the second main result of this section, and gives a non-recursive formula for the $D$-polynomials of a Coxeter group $W(X)$.

Theorem 3.7 Let $X$ be such that (2.4) holds. For all $x \in W_{c}(X)$ and $w \notin W_{c}(X)$ such that $x<w$, we have

$$
D_{x, w}(q)=\sum\left((-1)^{k} \prod_{i=1}^{k} P_{x_{i-1}, x_{i}}(q)\right),
$$


where the sum is over all the chains $x=x_{0}<x_{1}<\cdots<x_{k}=w$ such that $x_{i} \notin$ $W_{c}(X)$ if $i>0$, and $1 \leq k \leq \ell(x, w)$.

Proof We proceed by induction on $\ell(x, w)$.

If $\ell(x, w)=1$ then, from Lemma 3.6, we get $D_{x, w}(q)=-P_{x, w}(q)$, proving the claim in this case. If $\ell(x, w)>1$ then, from Lemma 3.6 and our induction hypothesis, we have

$$
\begin{aligned}
& D_{x, w}(q)=-P_{x, w}(q)-\sum_{\substack{t \notin W_{c}(X) \\
x<t<w}} D_{x, t}(q) P_{t, w}(q) \\
& =-P_{x, w}(q)-\sum_{\substack{t \notin W_{c}(X) \\
x<t<w}} P_{t, w}(q) \sum_{k=1}^{\ell(x, t)}\left(\sum_{x=x_{0}<\cdots<x_{k}=t}(-1)^{k} \prod_{i=1}^{k} P_{x_{i-1}, x_{i}}(q)\right) \\
& =-P_{x, w}(q)+\sum_{\substack{t \notin W_{c}(X) \\
x<t<w}}\left(\sum_{k=1}^{\ell(x, t)}\left(\sum_{\substack{x=x_{0}<\cdots<x_{k+1}=w \\
x_{k}=t}}(-1)^{k+1} \prod_{i=1}^{k+1} P_{x_{i-1}, x_{i}}(q)\right)\right) \\
& =\sum_{\substack{t \notin W_{c}(X) \\
x<t<w}}\left(\sum_{k \geq 0}\left(\sum_{\substack{x=x_{0}<\cdots<x_{k+1}=w, x_{k}=t \text { if } k \neq 0}}(-1)^{k+1} \prod_{i=1}^{k+1} P_{x_{i-1}, x_{i}}(q)\right)\right) \\
& =\sum_{k \geq 0}\left(\sum_{\substack{t \notin W_{c}(X) \\
x<t<w}}\left(\sum_{\substack{x=x_{0}<\cdots<x_{k+1}=w, x_{k}=t \text { if } k \neq 0}}(-1)^{k+1} \prod_{i=1}^{k+1} P_{x_{i-1}, x_{i}}(q)\right)\right) \\
& =\sum_{k \geq 0}\left(\sum_{\substack{x=x_{0}<\cdots \\
\cdots<x_{k+1}=w}}(-1)^{k+1} \prod_{i=1}^{k+1} P_{x_{i-1}, x_{i}}(q)\right) \text {, }
\end{aligned}
$$

and the result follows.

Theorem 3.7 shows that the $D$-polynomials are intimately related to the KazhdanLusztig polynomials, which is not at all obvious from their definition.

Lemma 3.8 Let $W(X)$ be a finite Coxeter group. Then

$$
x \in W_{c}(X) \Longleftrightarrow x^{-1} \in W_{c}(X) \quad \Longleftrightarrow w_{0} x w_{0} \in W_{c}(X),
$$

for all $x \in W(X)$.

Proof The maps $x \mapsto w_{0} x w_{0}$ and $x \mapsto x^{-1}$ are Bruhat order automorphisms (see [3, Proposition 2.3.4 and Corollary 2.3.6]). Moreover, both these maps send Coxeter generators to Coxeter generators, since $\ell(x)=\ell\left(x^{-1}\right)=\ell\left(w_{0} x w_{0}\right)$ (see $[3$, Corollary 2.3.3]). 
We now derive some consequences of Theorem 3.7. First we obtain some symmetry properties of the polynomials $\left\{D_{x, w}(q)\right\}_{x \in W_{c}(X), w \in W(X)}$.

Corollary 3.9 Let $x \in W_{c}(X), w \notin W_{c}(X)$ and $x<w$. Then we have

(i) $D_{x, w}(q)=D_{x^{-1}, w^{-1}}(q)$;

(ii) $D_{x, w}(q)=D_{w_{0} x w_{0}, w_{0} w w_{0}}(q)$.

Proof By Lemma 3.8, $x^{-1} \in W_{c}(X)$ for every $x \in W_{c}(X)$. Therefore we get

$$
\begin{aligned}
& D_{x^{-1}, w^{-1}}(q)= \sum_{\substack{x^{-1}=x_{0}<x_{1}<\cdots \\
\cdots<x_{k}=w^{-1}}}\left((-1)^{k} \prod_{i=1}^{k} P_{x_{i-1}, x_{i}}(q)\right) \\
&= \sum_{\substack{x=x_{0}^{-1}<x_{1}^{-1}<\cdots \\
\cdots<x_{k}^{-1}=w}}\left((-1)^{k} \prod_{i=1}^{k} P_{x_{i-1}, x_{i}}(q)\right) \\
&= \sum_{\substack{x=x_{0}^{-1}<x_{1}^{-1}<\cdots \\
\cdots<x_{k}^{-1}=w}}\left((-1)^{k} \prod_{i=1}^{k} P_{x_{i-1}^{-1}, x_{i}^{-1}}(q)\right) \\
&=\sum_{\substack{x=y_{0}<y_{1}<\cdots \\
\cdots<y_{k}=w}}\left((-1)^{k} \prod_{i=1}^{k} P_{y_{i-1}, y_{i}}(q)\right) . \\
&=D_{x, w}(q),
\end{aligned}
$$

where we have used a well known property of the Kazhdan-Lusztig polynomials (see, e.g., [3, §5, Exercise 12]). The same holds for $D_{w_{0} x w_{0}, w_{0} w w_{0}}(q)$, using Lemma 3.8 and the properties in $[3, \S 5$, Exercise 13$]$.

Next we compute the constant term of the polynomials $D_{x, w}(q)$.

Corollary 3.10 For all $x \in W_{c}(X)$ and $w \notin W_{c}(X)$ such that $x<w$, we have

$$
D_{x, w}(0)=\sum_{\substack{x=x_{0}<\cdots \\ \cdots<x_{k}=w}}(-1)^{k},
$$

where $x_{i} \notin W_{c}(X)$ if $i>0$, and $1 \leq k \leq \ell(x, w)$.

Proof The statement follows immediately from Theorem 3.7 and the well known fact that $P_{x, w}(0)=1$ for all $x, w \in W(X)$ such that $x \leq w$ (see, e.g., [3, Proposition 5.1.5]). 
By [47, Proposition 3.8.5], Corollary 3.10 asserts that $D_{x, w}(0)$ equals the Möbius function $\mu(\hat{0}, w)$ in the poset $\left\{y \in W(X) \backslash W_{c}(X): y \in[x, w]\right\} \cup\{\hat{0}\}$. This suggests the study of the partial order induced on $W(X) \backslash W_{c}(X)$ by the Bruhat order.

Lemma 3.11 Let $x \in W_{c}(X), w \notin W_{c}(X)$ be such that $x<w$. If $\ell(x, w)=1$ then $D_{x, w}(q)=-1$. If $\ell(x, w)=2$, then

$$
D_{x, w}(q)= \begin{cases}1 & \text { if } k=2, \\ 0 & \text { if } k=1, \\ -1 & \text { if } k=0,\end{cases}
$$

with $k \stackrel{\text { def }}{=}\left|\left\{y \notin W_{c}(X): x<y<w\right\}\right|$.

Proof By Lemma 2.4, $P_{x, w}(q)=1$ for all $x, w \in W(X)$ such that $\ell(x, w) \leq 2$. If $\ell(x, w)=1$, then Theorem 3.7 implies $D_{x, w}(q)=-P_{x, w}(q)=-1$. If $\ell(x, w)=$ 2 , then the interval $[x, w]$ is isomorphic to the boolean lattice $B_{2}$ (see, e.g., [3, Lemma 2.7.3]), so $k \in\{0,1,2\}$. Moreover, $P_{x, y}(q)=P_{y, w}(q)=1$ for all $y \notin W_{c}(X)$ such that $x<y<w$, since $\ell(x, y)=\ell(y, w)=1$. From Theorem 3.7 we get

$$
\begin{aligned}
D_{x, w}(q) & =\sum\left((-1)^{k} \prod_{i=1}^{k} P_{x_{i-1}, x_{i}}(q)\right) \\
& =-P_{x, w}(q)+\sum_{\substack{y \notin W_{c}(X) \\
x<y<w}} P_{x, y}(q) P_{y, w}(q) \\
& =-1+\sum_{\substack{y \notin W_{c}(X) \\
x<y<w}} 1 \\
& =-1+k,
\end{aligned}
$$

and the statement follows.

We end this section by deriving from Theorem 3.7 a closed formula for the polynomials $D_{x, w}(q)$ indexed by elements $x \in W_{c}(X)$ and $w \notin W_{c}(X)$ such that $\left([x, w] \cap\left(W(X) \backslash W_{c}(X)\right)\right) \cup\{x\}=[x, w] \cong B_{l(x, w)}$. In type $A$ it is easy to realize this case. Let $x \in W\left(A_{n}\right)$. Recall that $x$ is said to be a Coxeter element if $s_{\sigma(1)} \cdots s_{\sigma(n)}$ is a reduced expression for $x$, for some $\sigma \in S_{n}$. It is clear that a Coxeter element is always a fully commutative element.

Theorem 3.12 Let $s_{1} s_{2} \cdots s_{n} \cdots s_{2} s_{1}$ be a reduced expression for $w \in W\left(A_{n}\right)$ and let $x \in W\left(A_{n}\right)$ be a Coxeter element. Then the following hold:

(i) $x \leq w$;

(ii) $[x, w] \cong B_{\ell(x, w)}$;

(iii) $\left([x, w] \cap\left(W\left(A_{n}\right) \backslash W_{c}\left(A_{n}\right)\right)\right) \cup\{x\}=[x, w]$. 
Proof

(i) We find a reduced expression for $x$ which is a subexpression of

$s_{1} s_{2} \cdots s_{n} \cdots s_{2} s_{1}$

From [44, Theorem 1.5] there is a bijection between the set of Coxeter elements and the acyclic orientations of the Coxeter graph $A_{n}$. Let $A_{n}^{x}$ be the acyclic orientation of the graph $A_{n}$ associated to $x$. We say that $s_{i}$ is on the left (respectively, on the right) of $s_{i+1}$ in $x=s_{\sigma(1)} \cdots s_{\sigma(n)}$ if $s_{i} \longrightarrow s_{i+1}$ (respectively, $s_{i} \longleftarrow s_{i+1}$ ) in $A_{n}^{x}$. Therefore we are able to produce a reduced expression for $x$ from $A_{n}^{x}$ in the following way: set $x_{n}:=s_{n}$ and juxtapose $s_{n-1}$ to the left (respectively, to the right) of $x_{n}$ if $s_{n-1} \longrightarrow s_{n}$ (respectively, $s_{n-1} \longleftarrow s_{n}$ ). Set $x_{n-1}:=s_{n-1} x_{n}$ (respectively, $x_{n} s_{n-1}$ ). Repeat the same process with $x_{n-1}$ and $s_{n-2}$, and so on. The process ends when we get $x_{1}$. In fact, $x_{1}$ is a reduced expression for $x$ and $x_{1}$ is, by construction, a subexpression of $w$. Hence (i) follows from Theorem 3.3.

(ii) By Theorem 3.3, every element $y \in[x, w]$ admits (at least) one reduced expression that is a subexpression of $s_{1} s_{2} \cdots s_{n} \cdots s_{2} s_{1}$. Let $r(y)$ be one of these reduced expressions. Observe that the reduced expression $x_{1}$ obtained in (i) is a possible choice for $r(x)$. Consider the map $\phi:[x, w] \rightarrow \mathscr{A}$, with $\mathscr{A}=\left\{\left(\alpha_{1}, \ldots, \alpha_{n-1}\right)\right.$ : $\left.\alpha_{i} \in\{1,2\}\right\}$, such that $\phi(y)=\left(\alpha_{1}, \ldots, \alpha_{n-1}\right)$ if and only if $r(y)$ has $\alpha_{i}$ occurrences of the generator $s_{i}$. By [40, Corollary 3.3], the map $\phi$ is well-defined. We claim that $\phi$ is a bijection.

First, we prove the surjectivity. Fix $\left(\alpha_{1}, \ldots, \alpha_{n-1}\right) \in \mathscr{A}$. We describe an algorithm to construct (a reduced expression $r(y)$ for) an element $y \in W\left(A_{n}\right)$ such that $y \in[x, w]$ and $\phi(y)=\left(\alpha_{1}, \ldots, \alpha_{n-1}\right)$ in the following way: set $y_{n}:=s_{n}$. If $\alpha_{n-1}=2$ then set $y_{n-1}:=s_{n-1} y_{n} s_{n-1}$. Otherwise, proceed as in the proof of point (i), that is, juxtapose $s_{n-1}$ to the left (respectively, to the right) of $y_{n}$ if $s_{n-1} \longrightarrow s_{n}$ (respectively, $s_{n-1} \longleftarrow s_{n}$ ) and set $y_{n-1}:=s_{n-1} y_{n}$ (respectively, $y_{n} s_{n-1}$ ). Repeat the same process with $y_{n-1}$ and $\alpha_{n-2}$, and so on. The process ends when we get $y_{1}$. In fact, $r(x)=x_{1}$ is a subexpression of $y_{1}$ by construction. Next, we show that $y_{1}$ is a reduced expression. Observe that if $y_{j}$ is reduced then $y_{j} s_{j-1}>y_{j}$ and $s_{j-1} y_{j}>y_{j}$, since there is no occurrence of $s_{j-1}$ in $y_{j}$. Now, we proceed by contradiction to prove that $s_{j-1} y_{j} s_{j-1}$ is reduced. Suppose $\ell\left(s_{j-1} y_{j} s_{j-1}\right)<\ell\left(y_{j}\right)+2$, i.e., $\ell\left(s_{j-1} y_{j} s_{j-1}\right) \leq \ell\left(y_{j}\right)$. By applying Lemma 3.4 with $x=s_{j-1} y_{j} s_{j-1}$ and $w=y_{j} s_{j-1}$ we get $s_{j-1} y_{j}=y_{j} s_{j-1}$, and $s_{j-1} y_{j}, y_{j} s_{j-1}$ are both reduced expressions. Hence, [40, Lemma 3.1] implies that $s_{j-1}$ commutes with each generator in $y_{j}$, which is absurd, since $s_{j} \leq y_{j}$ by construction. Therefore

$$
\ell\left(s_{j-1} y_{j} s_{j-1}\right)=\ell\left(y_{j}\right)+2 .
$$

We conclude that $y_{1}$ is a reduced expression by induction on $n-i$, with $i=0 \cdots n-1$.

Denote by $y \in W\left(A_{n}\right)$ the element that admits $y_{1}$ as a reduced expression. Then $y$ has the desired properties.

For the injectivity we proceed by contradiction. Suppose that $u, v \in[x, w]$ are such that $\phi(u)=\phi(v)=\left(\alpha_{1}, \ldots, \alpha_{n-1}\right)$, with $u \neq v$. Denote by $r(u)$ (respectively, $r(v)$ ) the reduced expression of $u$ (respectively, $v$ ) obtained by applying the algorithm described above. Then $u \neq v$ implies $r(u) \neq r(v)$, that is, there exists an index $i \in$ 
[ $n-1]$ such that $\alpha_{i}=1$ and the position of the factor $s_{i}$ in $r(u)$ and $r(v)$ is different. Denote by $j$ be the minimum among these indices. Therefore, for every $h<j$ such that $\alpha_{h}=1, s_{h}$ appears on the same side in $r(u)$ as $r(v)$. Suppose that $\alpha_{j+1}=1$ and, for instance, that

$$
r(u)=y_{1} s_{j} \widehat{s_{j+1}} \cdots s_{n} \cdots s_{j+1} \widehat{s_{j}} y_{2}
$$

where $y_{1} \leq s_{1} s_{2} \cdots s_{j-1}$ and $y_{2} \leq s_{j-1} s_{j-2} \cdots s_{1}$. Then

$$
r(v)=y_{1} \widehat{s_{j}} s_{j+1} \cdots s_{n} \cdots \widehat{s_{j+1}} s_{j} y_{2} \quad \text { or } \quad r(v)=y_{1} \widehat{s_{j}} \widehat{s_{j+1}} \cdots s_{n} \cdots s_{j+1} s_{j} y_{2} .
$$

In both cases, $r(v)$ is a reduced expression such that $s_{j}$ is on the right of $s_{j+1}$. On the other hand, $r(u)$ is a reduced expression of $u$ such that $s_{j}$ is on the left of $s_{j+1}$. Hence, Theorem 3.3 implies that $x$ admits a reduced expression that is a subexpression of $r(u)$ and a (possibly different) reduced expression that is a subexpression of $r(v)$. This is a contradiction, since $x$ is uniquely determined by the relations $s_{i} \longrightarrow s_{i+1}$ or $s_{i} \longleftarrow s_{i+1}$. The same conclusion holds if we consider different deletions of $s_{j}$ and $s_{j+1}$. In the case $\alpha_{j+1}=2$, we may assume that

$$
r(u)=y_{1} s_{j} s_{j+1} \cdots s_{n} \cdots s_{j+1} \widehat{s_{j}} y_{2} \quad \text { and } \quad r(v)=y_{1} \widehat{s_{j}} s_{j+1} \cdots s_{n} \cdots s_{j+1} s_{j} y_{2} .
$$

Observe that $r(v)$ (respectively, $r(u)$ ) is a reduced expression such that $s_{j}$ is on the right (respectively, on the left) of $s_{j+1}$. Therefore, we reach the same contradiction that we obtained in the previous case.

(iii) Let $y \in(x, w]$ and $\phi(y)=\left(\alpha_{1}, \ldots, \alpha_{n-1}\right)$. Let $j$ be the maximum of the $i \in$ $[n-1]$ such that $\alpha_{i}=2$. Then $r(y)$ contains the substring $s_{j} s_{j+1} s_{j}$, so $y \notin W_{c}(X)$.

Corollary 3.13 Let $s_{1} s_{2} \cdots s_{n} \cdots s_{2} s_{1}$ be a reduced expression for $w \in W\left(A_{n}\right)$ and let $x \in W\left(A_{n}\right)$ be a Coxeter element. Then $D_{x, w}(q)=\varepsilon_{x} \varepsilon_{w}$.

Proof If $n=1$ then $x=w$ and the statement follows trivially. Suppose $n>1$. If $u, v \in W\left(A_{n}\right)$ are such that $[u, v] \simeq B_{\ell(u, v)}$, then $P_{u, v}(q)=1$ (see [6, Corollary 4.12]). Therefore, Theorem 3.12 implies that $P_{u, v}(q)=1$, for all $u, v \in[x, w]$. Hence, from Theorem 3.7 and Theorem 3.12 we achieve

$$
D_{x, w}(q)=\sum\left((-1)^{k} \prod_{i=1}^{k} P_{x_{i-1}, x_{i}}(q)\right)=\sum(-1)^{k},
$$

where the sum runs over all the chains $x=x_{0}<x_{1}<\cdots<x_{k}=w$ such that $1 \leq k \leq$ $\ell(x, w)$. Therefore, $D_{x, w}(q)$ equals the alternating sum

$$
\sum_{k=1}^{\ell(x, w)}(-1)^{k} c_{k}
$$

where $c_{k}$ denotes the number of chains $x=x_{0}<x_{1}<\cdots<x_{k}=w$. By [47, Proposition 3.8.5], we get

$$
\sum_{k=1}^{\ell(x, w)}(-1)^{k} c_{k}=\mu(x, w),
$$


where $\mu$ denotes the Möbius function on the poset induced by the Bruhat order on $[x, w]$. On the other hand, if $\mathscr{P}=(\mathscr{P}, \leq)$ is a boolean poset and $U, V \in \mathscr{P}$ are such that $U \leq V$, then $\mu_{\mathscr{P}}(U, V)=(-1)^{|V-U|}$, where $|V-U|$ denotes the length of the interval $[U, V]$ (see, e.g., [47, Example 3.8.3]). Finally, observe that the length of a Bruhat interval $[x, w]$ is $\ell(x, w)$ (see, e.g., [3, Theorem 2.2.6]) and the statement follows.

\section{The involution $\iota$}

In this section we study the family of polynomials $\left\{a_{x, w}(q)\right\}_{x, w \in W_{c}(X)} \subseteq \mathbb{Z}[q]$ which express the involution $\iota$ in terms of the $t$-basis (see Proposition 2.10). More precisely, we obtain, using the results in Sect. 3, a non-recursive formula for these polynomials, an expression for their constant term and symmetry properties.

Proposition 4.1 Let $X$ be such that (2.4) holds. Let $x, w \in W_{c}(X)$ be such that $x \leq$ $w$. Then

$$
a_{x, w}(q)=\varepsilon_{x} \varepsilon_{w} R_{x, w}(q)+\sum_{\substack{y \notin W_{c}(X) \\ x<y<w}} \varepsilon_{y} \varepsilon_{w} R_{y, w}(q)\left(\sum(-1)^{k} \prod_{i=1}^{k} P_{x_{i-1}, x_{i}}(q)\right),
$$

where the second sum runs over all the chains $x=x_{0}<\cdots<x_{k}=y$ such that $x_{i} \notin$ $W_{c}(X)$ if $i>0$.

Proof From Theorem 2.1 and Proposition 2.10 we get

$$
a_{x, w}(q)=\sum_{x \leq y \leq w} \varepsilon_{y} \varepsilon_{w} R_{y, w}(q) D_{x, y}(q)
$$

for all $x, w \in W_{c}(X)$ such that $x \leq w$. Since $D_{x, w}(q)=\delta_{x, w}$ if $x, w \in W_{c}(X)$, we have

$$
a_{x, w}(q)=\varepsilon_{x} \varepsilon_{w} R_{x, w}(q)+\sum_{\substack{y \notin W_{c}(X) \\ x<y<w}} \varepsilon_{y} \varepsilon_{w} R_{y, w}(q) D_{x, y}(q),
$$

so the statement follows immediately from Theorem 3.7.

Proposition 4.1 allows us to compute the constant term of the polynomial $a_{x, w}(q)$.

Corollary 4.2 For all $x, w \in W_{c}(X)$ such that $x \leq w$ we have

$$
a_{x, w}(0)=\sum(-1)^{k},
$$

where the sum runs over all the chains $x=x_{0}<x_{1}<\cdots<x_{k+1}=w$ such that $x_{i} \notin W_{c}(X)$ if $1 \leq i \leq k$, and $0 \leq k \leq \ell(x, w)-1$. 
Proof The statement follows from the fact that $R_{x, w}(0)=\varepsilon_{x} \varepsilon_{w}$ (see [3, Proposition 5.1.3]) and from Proposition 4.1.

Again, we deduce from Proposition 4.1 the following symmetry properties of the polynomials $\left\{a_{x, w}(q)\right\}_{x, w \in W_{c}(X)}$.

Corollary 4.3 Let $x, w \in W_{c}(X)$. Then we have

(i) $a_{x, w}(q)=a_{x^{-1}, w^{-1}}(q)$;

(ii) $a_{x, w}(q)=a_{w_{0} x w_{0}, w_{0} w w_{0}}(q)$.

Proof By Lemma 3.8 and by (4.1) we get

$$
\begin{aligned}
a_{x^{-1}, w^{-1}}(q) & =\sum_{x^{-1} \leq y \leq w^{-1}} \varepsilon_{y} \varepsilon_{w^{-1}} R_{y, w^{-1}}(q) D_{x^{-1}, y}(q) \\
& =\sum_{x^{-1} \leq z^{-1} \leq w^{-1}} \varepsilon_{z^{-1}} \varepsilon_{w^{-1}} R_{z^{-1}, w^{-1}}(q) D_{x^{-1}, z^{-1}}(q) \\
& =\sum_{x \leq z \leq w} \varepsilon_{z} \varepsilon_{w} R_{z, w}(q) D_{x, z}(q) \\
& =a_{x, w}(q),
\end{aligned}
$$

where we used Corollary 3.9(i) and the property $R_{x, w}(q)=R_{x^{-1}, w^{-1}}(q)$, for all $x, w \in W(X)$ (see, e.g., [3, §5, Exercise 10.(a)]). The same holds for $a_{w_{0} x w_{0}, w_{0} w w_{0}}(q)$, using Corollary 3.9(ii) and [3, §5, Exercise 10.(b)].

Lemma 4.4 Let $x, w \in W_{c}(X)$ be such that $x<w$. If $\ell(x, w)=1$ then $a_{x, w}(q)=$ $1-q$. If $\ell(x, w)=2$, then

$$
a_{x, w}(q)= \begin{cases}q^{2}-1 & \text { if } k=2, \\ q^{2}-q & \text { if } k=1, \\ q^{2}-2 q+1 & \text { if } k=0\end{cases}
$$

with $k \stackrel{\text { def }}{=}\left|\left\{y \notin W_{c}(X): x<y<w\right\}\right|$.

Proof By Lemma 2.4, $R_{x, w}(q)=(q-1)^{\ell(x, w)}$ for all $x, w \in W(X), x \leq w$ such that $\ell(x, w) \leq 2$. If $\ell(x, w)=1$, then (4.2) implies $a_{x, w}(q)=\varepsilon_{x} \varepsilon_{w} R_{x, w}(q)=1-q$. If $\ell(x, w)=2$, then the interval $[x, w]$ is isomorphic to the boolean lattice $B_{2}$ (see, e.g., [3, Lemma 2.7.3]). Moreover $R_{y, w}(q)=q-1$ and $D_{x, y}(q)=-1$ for all $y \notin$ $W_{c}(X)$ such that $x<y<w$, since $\ell(x, y)=\ell(y, w)=1$ (see Lemma 3.11). From Equation (4.2) we get

$$
a_{x, w}(q)=\varepsilon_{x} \varepsilon_{w} R_{x, w}(q)+\sum_{\substack{y \notin W_{c}(X) \\ x<y<w}} \varepsilon_{y} \varepsilon_{w} R_{y, w}(q) D_{x, y}(q)
$$




$$
\begin{aligned}
& =(q-1)^{2}+\sum_{\substack{y \notin W_{c}(X) \\
x<y<w}}(q-1) \\
& =(q-1)^{2}+k(q-1),
\end{aligned}
$$

and the statement follows.

\section{The polynomials $L_{x, w}$}

In this section we study the polynomials $\left\{L_{x, w}\left(q^{-\frac{1}{2}}\right)\right\}_{x, w \in W_{c}(X)}$ which play the same role, in $T L(X)$, as the Kazhdan-Lusztig polynomials in $\mathscr{H}(X)$. More precisely, using the results in the previous section, we obtain a non-recursive formula, symmetry properties and expressions for the constant term for these polynomials.

Theorem 5.1 Let $X$ be such that (2.4) holds. For all elements $x, w \in W_{c}(X)$ such that $x<w$ we have

$$
L_{x, w}\left(q^{-\frac{1}{2}}\right)=q^{\frac{\ell(x)-\ell(w)}{2}} \sum\left((-1)^{k} \prod_{i=1}^{k+1} P_{x_{i-1}, x_{i}}(q)\right),
$$

where the sum runs over all the chains $x=x_{0}<x_{1}<\cdots<x_{k+1}=w$ such that $x_{i} \notin W_{c}(X)$ if $1 \leq i \leq k$, and $0 \leq k \leq \ell(x, w)-1$.

Proof On the one hand, from Proposition 2.8 and the definition of the $t$-basis we get

$$
\begin{aligned}
\sigma\left(C_{w}^{\prime}\right) & =q^{-\frac{\ell(w)}{2}} \sum_{y \leq w} P_{y, w}(q) \sigma\left(T_{y}\right) \\
& =q^{-\frac{\ell(w)}{2}} \sum_{y \leq w} P_{y, w}(q)\left(\sum_{\substack{x \in W_{c}(X) \\
x \leq y}} D_{x, y}(q) t_{x}\right) \\
& =q^{-\frac{\ell(w)}{2}} \sum_{\substack{x \in W_{c}(X) \\
x \leq w}}\left(\sum_{x \leq y \leq w} D_{x, y}(q) P_{y, w}(q)\right) t_{x} .
\end{aligned}
$$

On the other hand, by definition,

$$
c_{w}=\sum_{\substack{x \in W_{c}(X) \\ x \leq w}} q^{-\frac{\ell(x)}{2}} L_{x, w}\left(q^{-\frac{1}{2}}\right) t_{x} .
$$

Therefore, by (2.4),

$$
q^{-\frac{\ell(x)}{2}} L_{x, w}\left(q^{-\frac{1}{2}}\right)=q^{-\frac{\ell(w)}{2}} \sum_{x \leq y \leq w} D_{x, y}(q) P_{y, w}(q) .
$$


Since $D_{x, y}(q)=\delta_{x, y}$ if $y \in W_{c}(X)$ and $x \leq y$, we achieve

$$
L_{x, w}\left(q^{-\frac{1}{2}}\right)=q^{\frac{\ell(x)-\ell(w)}{2}}\left(P_{x, w}(q)+\sum_{\substack{y \notin W_{c}(X) \\ x<y<w}} D_{x, y}(q) P_{y, w}(q)\right) .
$$

Combining (5.1) and Theorem 3.7 we get

$$
\begin{aligned}
& L_{x, w}\left(q^{-\frac{1}{2}}\right) \\
& =q^{\frac{\ell(x)-\ell(w)}{2}}\left(P_{x, w}(q)+\sum_{\substack{y \notin W_{c}(X) \\
x<y<w}} \sum_{\substack{x=x_{0}<\cdots<\\
\cdots<x_{k}=y}}\left((-1)^{k} \prod_{i=1}^{k} P_{x_{i-1}, x_{i}}(q)\right) P_{y, w}(q)\right) \\
& =q^{\frac{\ell(x)-\ell(w)}{2}}\left(\sum_{\substack{x=x_{0}<\cdots \\
\cdots x_{k+1}=w}}(-1)^{k} \prod_{i=1}^{k+1} P_{x_{i-1}, x_{i}}(q)\right),
\end{aligned}
$$

where $x_{i} \notin W_{c}(X)$ if $1 \leq i \leq k$, as desired.

Theorem 5.1 shows that the $L$-polynomials depend only on the Kazhdan-Lusztig polynomials and the poset structure induced by the Bruhat order on $\{x, w\} \cup((x, w) \backslash$ $\left.(x, w)_{c}\right)$, where $(x, w)_{c}=\left\{y \in(x, w): y \in W_{c}(X)\right\}$.

In the same way that Corollary 3.9 follows from Theorem 3.7 we deduce from Theorem 5.1 the following symmetry properties of the $L$-polynomials, whose proof we omit.

Corollary 5.2 For all $x, w \in W_{c}(X)$ such that $y \leq w$, we have

(i) $L_{x, w}\left(q^{-\frac{1}{2}}\right)=L_{x^{-1}, w^{-1}}\left(q^{-\frac{1}{2}}\right)$;

(ii) $L_{x, w}\left(q^{-\frac{1}{2}}\right)=L_{w_{0} x w_{0}, w_{0} w w_{0}}\left(q^{-\frac{1}{2}}\right)$.

Lemma 5.3 Let $x, w \in W_{c}(X)$ be such that $x<w$. If $\ell(x, w)=1$ then $L_{x, w}\left(q^{-\frac{1}{2}}\right)=$ $q^{-\frac{1}{2}}$. If $\ell(x, w)=2$, then

$$
L_{x, w}\left(q^{-\frac{1}{2}}\right)= \begin{cases}-q^{-1} & \text { if } k=2, \\ 0 & \text { if } k=1 \\ q^{-1} & \text { if } k=0\end{cases}
$$

with $k \stackrel{\text { def }}{=}\left|\left\{y \notin W_{c}(X): x<y<w\right\}\right|$.

Proof If $\ell(x, w)=1$, then $P_{x, w}(q)=1$ (see Lemma 2.4). Equation (5.1) then implies that $L_{x, w}\left(q^{-\frac{1}{2}}\right)=q^{-\frac{1}{2}} P_{x, w}(q)=q^{-\frac{1}{2}}$. If $\ell(x, w)=2$, then from (5.1) and Lemma 3.11 we get

$$
L_{x, w}\left(q^{-\frac{1}{2}}\right)=q^{\frac{\ell(x)-\ell(w)}{2}}\left(P_{x, w}(q)+\sum_{\substack{y \notin W_{c}(X) \\ x<y<w}} D_{x, y}(q) P_{y, w}(q)\right)
$$




$$
\begin{aligned}
& =q^{-1}\left(1+\sum_{\substack{y \notin W_{c}(X) \\
x<y<w}}(-1)\right) \\
& =q^{-1}(1-k),
\end{aligned}
$$

and the statement follows.

The previous calculations show that $\left\{L_{x, w}\left(q^{-\frac{1}{2}}\right)\right\} \nsubseteq \mathbb{N}\left[q^{-\frac{1}{2}}\right]$.

Acknowledgements I would like to thank Prof. Francesco Brenti for introducing me to this topic and for many useful conversations.

\section{References}

1. Billey, S.C., Jockusch, W., Stanley, R.P.: Some combinatorial properties of Schubert polynomials. J. Algebr. Comb. 2, 345-374 (1993)

2. Billey, S.C., Warrington, G.S.: Kazhdan-Lusztig polynomials for 321-hexagon-avoiding permutations. J. Algebr. Comb. 13, 111-136 (2001)

3. Björner, A., Brenti, F.: Combinatorics of Coxeter Groups. Graduate Texts in Mathematics, vol. 231. Springer, Berlin (2005)

4. Boe, B.D.: Kazhdan-Lusztig polynomials for Hermitian symmetric spaces. Trans. Am. Math. Soc. 309, 279-294 (1988)

5. Brenti, F.: A combinatorial formula for Kazhdan-Lusztig polynomials. Invent. Math. 118, 371-394 (1994)

6. Brenti, F.: Combinatorial properties of the Kazhdan-Lusztig $R$-polynomials for $S_{n}$. Adv. Math. 126, 21-51 (1997)

7. Brenti, F.: Lattice paths and Kazhdan-Lusztig polynomials. J. Am. Math. Soc. 11, 229-259 (1998)

8. Brenti, F., Caselli, F., Marietti, M.: Special matchings and Kazhdan-Lusztig polynomials. Adv. Math. 202, 555-601 (2006)

9. Brenti, F., Simion, R.: Explicit formulae for some Kazhdan-Lusztig polynomials. J. Algebr. Comb. 11, 187-196 (2000)

10. Caselli, F.: Proof of two conjectures of Brenti and Simion on Kazhdan-Lusztig polynomials. J. Algebr. Comb. 18, 171-187 (2003)

11. Cautis, S., Jackson, D.M.: The matrix of chromatic joins and the Temperley-Lieb algebra. J. Comb. Theory, Ser. A 89, 109-155 (2003)

12. Delanoy, E.: Combinatorial invariance of Kazhdan-Lusztig polynomials on intervals starting from the identity. J. Algebr. Comb. 24, 437-463 (2006)

13. Deodhar, V.V.: On some geometric aspects of Bruhat orderings. II. The parabolic analogue of Kazhdan-Lusztig polynomials. J. Algebra 111, 483-506 (1987)

14. Deodhar, V.V.: A combinatorial setting for questions in Kazhdan-Lusztig theory. Geom. Dedic. 36, 95-119 (1990)

15. Du, J.: Global IC bases for quantum linear groups. J. Pure Appl. Algebra 114, $25-37$ (1996)

16. Dyer, M.J.: On coefficients of $q$ in Kazhdan-Lusztig polynomials. In: Algebraic groups and Lie groups. Austral. Math. Soc. Lect. Ser., vol. 9, pp. 189-194 (1997)

17. Fan, C.K.: A Hecke algebra quotient and properties of commutative elements of a Weyl group. Ph.D. thesis, M.I.T (1995)

18. Fan, C.K.: A Hecke algebra quotient and some combinatorial applications. J. Algebr. Comb. 5, 175189 (1996)

19. Fan, C.K., Green, R.M.: Monomials and Temperley-Lieb algebras. J. Algebra 190, 498-517 (1997)

20. Graham, J.J.: Modular representations of Hecke algebras and related algebras. Ph.D. thesis, University of Sydney (1995)

21. Green, R.M.: Generalized Temperley-Lieb algebras and decorated tangles. J. Knot Theory Ramif. 7, 155-171 (1998) 
22. Green, R.M.: Generalized Jones traces and Kazhdan-Lusztig bases. J. Pure Appl. Algebra 211, 744$772(2007)$

23. Green, R.M.: Leading coefficients of Kazhdan-Lusztig polynomials and fully commutative elements. J. Algebr. Comb. 30, 165-171 (2009)

24. Green, R.M., Losonczy, J.: Canonical bases for Hecke algebra quotients. Math. Res. Lett. 6, 213-222 (1999)

25. Green, R.M., Losonczy, J.: A projection property for Kazhdan-Lusztig bases. Int. Math. Res. Not. 1, $23-34(2000)$

26. Green, R.M., Losonczy, J.: Fully commutative Kazhdan-Lusztig cells. Ann. Inst. Fourier 51, 1025$1045(2001)$

27. Humphreys, J.E.: Reflection Groups and Coxeter Groups. Cambridge Studies in Advanced Mathematics, vol. 29. Cambridge University Press, Cambridge (1990)

28. Incitti, F.: On the combinatorial invariance of Kazhdan-Lusztig polynomials. J. Comb. Theory, Ser. A 113, 1332-1350 (2006)

29. Jones, B.C.: Leading coefficients of Kazhdan-Lusztig polynomials for Deodhar elements. J. Algebr. Comb. 29, 229-260 (2009)

30. Jones, V.F.R.: A polynomial invariant for knots via von Neumann algebras. Bull. Am. Math. Soc. 12, 103-111 (1985)

31. Jones, V.F.R.: Hecke algebra representations of braid groups and link polynomials. Ann. Math. 126, 335-388 (1987)

32. Kauffman, L., Thomas, R.: Temperley-Lieb algebras and the four-color theorem. Combinatorica 23, 653-667 (2003)

33. Kazhdan, D., Lusztig, G.: Representations of Coxeter groups and Hecke algebras. Invent. Math. 53, 165-184 (1979)

34. Lascoux, A.: Polynômes de Kazhdan-Lusztig pour les variétés de Schubert vexillaires. C. R., Math. 321, 667-670 (1995)

35. Lascoux, A., Schützenberger, M.-P.: Polynômes de Kazhdan \& Lusztig pour les grassmanniennes. Young tableaux and Schur functors in algebra and geometry (Toruń, 1980) 87, 249-266

36. Lascoux, A., Schützenberger, M.-P.: Treillis et bases des groupes de Coxeter. Electron. J. Combin. 3, R27 (1996)

37. Levy, D.: Structure of Temperley-Lieb algebras and its application to 2D statistical models. Phys. Rev. Lett. 64, 499-502 (1990)

38. Losonczy, J.: The Kazhdan-Lusztig basis and the Temperley-Lieb quotient in type D. J. Algebra 233, $1-15(2000)$

39. Marietti, M.: Closed product formulas for certain $R$-polynomials. Eur. J. Comb. 23, 57-62 (2002)

40. Marietti, M.: Boolean elements in Kazhdan-Lusztig theory. J. Algebra 295, 1-26 (2006)

41. Martin, P.P.: Temperley-Lieb algebra, group theory and the Potts model. J. Phys. A 3, 577-591 (1988)

42. Martin, P.P.: Temperley-Lieb algebras and the long distance properties of statistical mechanical models. J. Phys. A 23, 7-30 (1990)

43. Polo, P.: Construction of arbitrary Kazhdan-Lusztig polynomials in symmetric groups. Represent. Theory 3, 90-104 (1999)

44. Shi, J.-Y.: The enumeration of Coxeter elements. J. Algebr. Comb. 6, 161-171 (1997)

45. Shi, J.-Y.: Fully commutative elements and Kazhdan-Lusztig cells in the finite and affine Coxeter groups. Proc. Am. Math. Soc. 131, 3371-3378 (2003)

46. Shi, J.-Y.: Fully commutative elements and Kazhdan-Lusztig cells in the finite and affine Coxeter groups. II. Proc. Am. Math. Soc. 133, 2525-2531 (2005)

47. Stanley, R.P.: Enumerative Combinatorics, Vol. 1. Cambridge Studies in Advanced Mathematics, vol. 49. Cambridge University Press, Cambridge (1997)

48. Stembridge, J.R.: On the fully commutative elements of Coxeter groups. J. Algebr. Comb. 5, 353-385 (1996)

49. Temperley, H.N.V., Lieb, E.H.: Relations between the "percolation" and "colouring" problem and other graph-theoretical problems associated with regular planar lattices: some exact results for the “percolation” problem. Proc. R. Soc. Lond. Ser. A, Math. Phys. Sci. 322, 251-280 (1971) 